\title{
Is There a General Area Theorem for Black Holes?
}

\author{
Domenico Giulini \\ Institut für theoretische Physik, Universität Zürich \\ Winterthurerstrasse 190, CH-8057 Zürich, Switzerland \\ e-mail: giulini@physik.unizh.ch
}

\begin{abstract}
The general validity of the area law for black holes is still an open problem. We first show in detail how to complete the usually incompletely stated text-book proofs under the assumption of piecewise $C^{2}$-smoothness for the surface of the black hole. Then we prove that a black hole surface necessarily contains points where it is not $C^{1}$ (called "cusps") at any time before caustics of the horizon generators show up, like e.g. in merging processes. This implies that caustics never disappear in the past and that black holes without initial cusps will never develop such. Hence black holes which will undergo any non-trivial processes anywhere in the future will always show cusps. Although this does not yet imply a strict incompatibility with piecewise $C^{2}$ structures, it indicates that the latter are likely to be physically unnatural. We conclude by calling for a purely measure theoretic proof of the area theorem.
\end{abstract}

\section{Introduction}

It seems to be widely accepted as fact that the surface area of a black hole cannot decrease with time. However, the proofs offered in standard text-books, like [HE], $[\mathrm{MTW}]$ and $[\mathrm{W}]$, are basically content with the remark that this law follows from the non-convergence of the generators of the future event horizon. It would indeed follow from this remark and some elementary differential geometric considerations if the horizon were a sufficiently smooth submanifold. Mathematically there is absolutely no reason why this should be true in general [CG], which means that extra assumptions must be invoked (implicitly) in the text-books arguments. However, not much precise information seems to exist in the literature concerning these extra assumptions. Perhaps the clearest statement is given in $[\mathrm{CG}]$, where the authors mention that in the text-book proofs of the area theorem "something close to $C^{2}$ differentiability 'almost everywhere' of the event horizon seems to have been assumed". The text-books themselves do to mention any such condition. Below we 
show explicitly how to complete the text-book argumentation under the assumption of piecewise $C^{2}$-smoothness. But this clearly does not imply its necessity.

General considerations only prove the horizon to be locally Lipschitz continuous (denoted by $C^{1-}$ ) [HE]. Mathematically this implies (pointwise) differentiability almost everywhere (with respect to the Lebesgue measures defined by the charts, see $[\mathrm{F}]$ Theorem 3.1.6), but it still allows the points of non-differentiability to be densely distributed [CG]. Hence horizons exist which are nowhere $C^{1}$. Given these mathematical facts, it is of interest to learn what physical conditions imply a breakdown of $C^{1}$ differentiability. We prove that a black hole whose surface is $C^{1}$ at one time can never merge with other black holes and, more generally, never encounter new null generators for its future event horizon. In other words, an initial $C^{1}$-condition basically rules out any interesting physical process to happen in the future. Hence the only dynamically interesting holes for which the area law is actually proven are those whose surfaces are piecewise $C^{2}$ but not $C^{1}$. Presumably this class does not contain many, if any, physically realistic members. Cusps on the surfaces of colliding black holes can be clearly seen in numerical studies [L-W], but no analytic proof of their general existence seems to have been given so far.

Given the widely believed connection of the area law with thermodynamic properties of black holes on one side, and the widely expressed hope that this connection may be of heuristic value in understanding certain aspects of quantum gravity on the other, it seems important to know the most general conditions under which the area law is valid.

\section{Notation, Facts and Assumptions}

We assume space-time $(M, g)$ to be strongly asymptotically predictable (in the sense of $[\mathrm{W}]$ ) and globally hyperbolic. (It would be sufficient to restrict to a globally hyperbolic portion, as in Thm. 12.2.6 of [W].) $\mathcal{I}^{+}$(scri-plus) denotes future null infinity, $J^{-}\left(\mathcal{I}^{+}\right)$its causal past and $B:=M-J^{-}\left(\mathcal{I}^{+}\right)$the black-hole region. Its boundary, $\partial B=: H$, is the future-event-horizon. $H$ is a closed, imbedded, achronal three-dimensional $C^{1-}$-submanifold of $M$ (Proposition 6.3.1 in [HE]). $H$ is generated by null geodesics without future-endpoints. Past-endpoints occur only where null geodesics - necessarily coming from $J^{-}\left(\mathcal{I}^{+}\right)$- join onto $H$. Such points are called "caustics". Only at a caustic can a point of the horizon be intersected by more than 
one generator, and all generators that intersect a caustic enter the horizon at this point. Once a null geodesic has joined onto $H$ it will never encounter a caustic again (i.e. not intersect another generator) and never leave $H$. See Box 34.1 in [MTW] for a lucid discussion and partial proofs of these statements. Hence there are two different processes through which the area of a black hole may increase: First, new generators can join the horizon and, second, the already existing generators can mutually diverge.

Caustic points where $n$ (possibly infinite) new generators join in are said to be of multiplicity $n$. In $[\mathrm{BK}]$ it is proven that $H$ is not differentiable at $p$ iff $p$ is a caustic of multiplicity $n \geq 2$, and that caustics of multiplicity 1 are contained in the closure of those of higher multiplicity. In particular, $H$ cannot be of class $C^{1}$ at any caustic point. Points where $H$ is not $C^{1}$ will be called "cusps". By definition, being $C^{1}$ at $p$ implies that $H$ is differentiable in a whole neighbourhood $U$ of $p$. Conversely, it was shown that differentiability in some open neighbourhood $U$ of $p$ implies that $H$ is $C^{1}$ in $U$ ([BK], Prop. 3.3), so that the set of points where $H$ is $C^{1}$ is open. It follows that being $C^{1}$ at $p$ is in fact equivalent to being differentiable in some neighbourhood of $p$. Hence the set of cusps is the closure of the set of points where $H$ is non-differentiable (caustics of multiplicity $\geq 2$ ) and hence also the closure of the set of all caustics.

Let $\Sigma$ be a suitably smooth (usually $C^{2}$ ) Cauchy surface, then $\mathcal{B}:=B \cap \Sigma$ is called the black-hole region at time $\Sigma$ and $\mathcal{H}:=H \cap \Sigma=\partial \mathcal{B}$ the (future-event-) horizon at time $\Sigma$. A connected component $\mathcal{B}_{i}$ of $\mathcal{B}$ is called a black-hole at time $\Sigma$. Its surface is $\mathcal{H}_{i}=\partial B_{i}$, which is a two-dimensional, imbedded $C^{1-}$-submanifold of $\Sigma$. We have seen that in general $\mathcal{H}$ may contain all kinds of singularities which would render standard differential geometric methods inapplicable. Adding the hypothesis of piecewise $C^{2}$-smoothness circumvents this problem.

By exp :TM $T M$ we denote the exponential map. Recall that $\exp _{p}(v):=$ $\gamma(1)$, where $\gamma$ is the unique geodesic with initial conditions $\gamma(0)=p \in M$ and $\dot{\gamma}(0)=v \in T_{p}(M)$. For each $p$ it is well defined for $v$ in some open neighbourhood of $0 \in T_{p}(M)$. One has $\gamma(t)=\exp _{p}(t v)$. We shall assume the Lorentzian metric $g$ of $M$ to be $C^{2}$, hence the connection (i.e. the Christoffel Symbols) is $C^{1}$ and therefore the map exp is also $C^{1}$. The last assertion is e.g. proven in [L]. 


\section{Local Formulation of the Area Law}

We consider two $C^{2}$ Cauchy surfaces with $\Sigma^{\prime}$ to the future of $\Sigma$. The corresponding black-hole regions and surfaces are denoted as above, with a prime distinguishing those on $\Sigma^{\prime}$. We make the assumption that $\mathcal{H}$ is piecewise $C^{2}$, i.e. each connected component $\mathcal{H}_{i}$ of $\mathcal{H}$ is the union of open subsets $\mathcal{H}_{i}^{k}$ which are $C^{2}$ submanifolds of $M$ and whose 2-dimensional measure exhaust that of $\mathcal{H}_{i}: \mu\left(\mathcal{H}_{i}-\bigcup_{k} \mathcal{H}_{i}^{k}\right)=0$, where $\mu$ is the measure on $\mathcal{H}$ induced from the metric $g$.

For each point $p \in \mathcal{H}_{i}^{k}$ there is a unique future- and outward-pointing null direction perpendicular to $\mathcal{H}_{i}^{k}$, which we generate by some future directed $l(p) \in T_{p}(M)$. We can choose a $C^{1}$-field $p \mapsto l(p)$ of such vectors over $\mathcal{H}_{i}^{k}$. The geodesics $\gamma_{p}: t \mapsto \gamma_{p}(t):=\exp _{p}(t l(p))$ are generators of $H$ without future-endpoint. Therefore each $\gamma_{p}$ cuts $\Sigma^{\prime}$ in a unique point $p^{\prime} \in \mathcal{H}^{\prime}$ at a unique parameter value $t=\tau(p)$. By appropriately choosing the affine parametrisations of $\gamma_{p}$ as $p$ varies over $\mathcal{H}_{i}^{k}$ we can arrange the map $\tau$ to be also $C^{1}$. Hence $p \mapsto m(p):=\tau(p) l(p)$ is a null vector field of class $C^{1}$ over $\mathcal{H}_{i}^{k}$. We can now define the map

$$
\Phi_{i}^{k}: \mathcal{H}_{i}^{k} \rightarrow \mathcal{H}^{\prime}, \quad p \mapsto \Phi_{i}^{k}(p):=\exp _{p}(m(p)),
$$

which satisfies the following

Lemma 1. $\Phi_{i}^{k}$ is (i) $C^{1}$, (ii) injective, (iii) non-measure-decreasing.

(i) follows from the fact that the functions $m$ and $\exp$ are $C^{1}$. Injectivity must hold, since otherwise some of the generators of $H$ through $\mathcal{H}_{i}^{k}$ would cross in the future. By non-measure-decreasing we mean the following: Let $\mu$ and $\mu^{\prime}$ be the measures on $\mathcal{H}$ and $\mathcal{H}^{\prime}$ induced by the space-time metric $g$. Then $\mu[U] \leq \mu^{\prime}\left[\Phi_{i}^{k}(U)\right]$ for each measurable $U \subset \mathcal{H}_{i}^{k}$. Assuming the weak energy condition, this is a consequence of the nowhere negative divergence for the future geodesic congruence $p \mapsto \gamma_{p}$ (Lemma 9.2.2 in [HE]), as we will now show.

Proof of (iii): Set $H_{i}^{k}:=\bigcup_{p, t} \exp _{p}(t l(p)), \forall p \in \mathcal{H}_{i}^{k}$ and $\forall t \in R_{+}$, which is a $C^{1}$ submanifold of $M$ (the future of $\mathcal{H}_{i}^{k}$ in $H$ ). Let $l$ be the unique (up to a constant scale) future directed null geodesic (i.e. $\nabla_{l} l=0$ ) vector field on $H_{i}^{k}$ parallel to the generators. Then $0 \leq \nabla_{\mu} l^{\mu}=\pi_{\nu}^{\mu} \nabla_{\mu} l^{\nu}$, where $\pi$ denotes the map given by the $g$ orthogonal projection $\left.T(M)\right|_{H_{i}^{k}} \rightarrow T\left(H_{i}^{k}\right)$, followed by the quotient map $T\left(H_{i}^{k}\right) \rightarrow$ $T\left(H_{i}^{k}\right) / \operatorname{span}\{l\}$. Note that tangent spaces of $C^{1}$-cross-sections of $H_{i}^{k}$ at the point 
$p$ are naturally identified with $T_{p}\left(H_{i}^{k}\right) / \operatorname{span}\{l(p)\}$. Since $\pi_{\nu}^{\mu} l^{\nu}=0$, we also have $\pi_{\nu}^{\mu} \nabla_{\mu} k^{\nu} \geq 0$ for $k=\lambda l$ and any $C^{1}$-function $\lambda: H_{i}^{k} \rightarrow \mathbf{R}_{+}$. Hence this inequality is valid for any future pointing $C^{1}$-vector-field $k$ on $H_{i}^{k}$ parallel to the generators. Given that, let $t \mapsto \phi_{t}$ be the flow of $k$ and $A(t):=\mu_{t}\left[\phi_{t}(U)\right]:=\int_{\phi_{t}(U)} d \mu_{t}$, then $\dot{A}(t)=\int_{\phi_{t}(U)} \pi_{\nu}^{\mu}(t) \nabla_{\mu} k^{\nu}(t) d \mu_{t} \geq 0$, where $\pi(t)$ projects onto $T\left(\phi_{t}\left(\mathcal{H}_{i}^{k}\right)\right)$, $k(t)=\left.\frac{d}{d t^{\prime}}\right|_{t^{\prime}=t} \phi_{t^{\prime}}$ and $\mu_{t}=$ measure on $\phi_{t}\left(\mathcal{H}_{i}^{k}\right)$. Now choose $k$ such that $\phi_{t=1}=\Phi_{i}^{k}$, then $\mu^{\prime}\left[\Phi_{i}^{k}(U)\right]-\mu[U]=\int_{0}^{1} d t \dot{A}(t) \geq 0$.

Part (iii) of Lemma 1 is the local version of the area law. By turning it into a global statement about areas one usually abandons some of its information. The most trivial global implication is that the total sum of areas cannot decrease. A more refined version is as follows: Recall that black holes cannot bifurcate in the future (Proposition 9.2.5 of [HE]). Hence all surface elements $\mathcal{H}_{i}^{k}$ of the $i$-th blackhole at time $\Sigma$ are mapped via $\Phi_{i}^{k}$ into the surface $\mathcal{H}^{\prime}{ }_{i}$ of a single black-hole at time $\Sigma^{\prime}$. We call $\mathcal{H}^{\prime}{ }_{i}$ (i.e. the connected component of $\Sigma^{\prime} \cap H$ into which $\mathcal{H}_{i}$ is mapped) the development of $\mathcal{H}_{i}$ at time $\Sigma^{\prime}$. Lemma 1 now implies that its area cannot be less than that of $\mathcal{H}_{1}$. The non-bifurcation result implies that if the number $N^{\prime}$ of black holes at time $\Sigma^{\prime}$ is bigger than the number $N$ at time $\Sigma$, then there is an intermediate formation of $K \geq N^{\prime}-N$ new black holes. That these black-holes are 'new', i.e. not present at time $\Sigma$, means that all generators of $H$ which intersect $\mathcal{H}^{\prime}{ }_{1} \cup \cdots \cup \mathcal{H}^{\prime}{ }_{K}$ must have past-endpoints somewhere between $\Sigma$ and $\Sigma^{\prime}$. A black hole at time $\Sigma^{\prime}$ which is smaller than any black hole at time $\Sigma$ must also be new in this sense. Hence one way to express an area law would be as follows:

Assertion (Area Law). Consider two Cauchy surfaces, $\Sigma$ and $\Sigma^{\prime}$, with $\Sigma^{\prime}$ to the future of $\Sigma$. Then the area of the development $\mathcal{H}^{\prime}{ }_{i}$ of any $\mathcal{H}_{i}$ cannot be less than that of $\mathcal{H}_{i}$. In particular, black holes at time $\Sigma^{\prime}$ whose area is smaller than that of any black hole at time $\Sigma$ must have been formed in the meantime.

Presently we do not have a proof that this statement is true in general. But since it is a statement about measures, we suggest that it should be possible to give a proof without invoking fiducial (and probably irrelevant) differentiability assumptions. 


\section{Consequences}

The foregoing discussion allows to show that black holes whose surface is $C^{1}$ at one instant cannot undergo any non-trivial change in the future, like merging processes or any other process involving the incorporation of new generators. This we shall now explain in more detail. Let $\mathcal{H}_{1}$ be the surface a black hole at time $\Sigma$. We assume $\mathcal{H}_{1}$ to be a compact, 2 -dimensional $C^{1}$-submanifold. As before, $\Sigma^{\prime}$ is to the future of $\Sigma$ and $\mathcal{H}^{\prime}=H \cap \Sigma^{\prime}$. We can construct a map $\Phi_{1}: \mathcal{H}_{1} \rightarrow \mathcal{H}^{\prime}$, just analogous to the construction of $\Phi_{i}^{k}$ above, but now defined on all of $\mathcal{H}_{1}$. The $C^{1}$-condition on $\mathcal{H}_{1}$ now implies that $\Phi_{1}$ is $C^{0}$. $\Phi_{1}$ is also injective for the same reason as given for $\Phi_{i}^{k}$. Since $\mathcal{H}_{1}$ is connected, its image under $\Phi_{1}$ is also connected. Let $\mathcal{H}^{\prime}{ }_{1} \subset \mathcal{H}^{\prime}$ be the connected part containing the image of $\Phi_{1}$. We show

Lemma 2. $\Phi_{1}: \mathcal{H}_{1} \rightarrow \mathcal{H}^{\prime}{ }_{1}$ is a homeomorphism.

Proof. $\Phi_{1}$ is a closed map, because if $U \subset \mathcal{H}_{1}$ is closed $\Rightarrow U$ is compact (since $\mathcal{H}_{1}$ is compact) $\Rightarrow U^{\prime}:=\Phi_{1}(U)$ is compact (since $\Phi_{1}$ is continuous) $\Rightarrow U^{\prime}$ is closed ( since $\mathcal{H}^{\prime}{ }_{1}$ is Hausdorff). From this follows that $\Phi_{1}$ is a homeomorphism onto its image. But $\Phi_{1}$ is also open. This follows directly from Brouwer's theorem on the invariance-of-domain, which states that any continuous injective map from an open $X \subset R^{n}$ into $R^{n}$ is open (Proposition 7.4 in [D]). This clearly generalizes to manifolds. Hence the image $\Phi_{1}\left(\mathcal{H}_{1}\right) \subset \mathcal{H}^{\prime}{ }_{1}$ is open, closed and connected, and hence all of $\mathcal{H}^{\prime}{ }_{1}$.

Surjectivity of $\Phi_{1}$ implies that all generators of $H$ which intersect $\mathcal{H}^{\prime}{ }_{1}$ also intersect $\mathcal{H}_{1}$. Hence nowhere in its future will $\mathcal{H}_{1}$ be joined by new generators.

Similar to the definition of $H_{i}^{k}$ above, let $H_{1} \subset H$ denote the future of $\mathcal{H}_{1}$ in $H$; then it follows that $H_{1}$ is free of caustics and therefore $C^{1}$. Hence we have

Proposition 1. Let the surface of a black hole at time $\Sigma$ be without cusps. Then this black hole will never encounter cusps to the future of $\Sigma$, in particular, it will not merge with other black holes.

Another equivalent formulation, emphasizing that cusps will not die out in the past, is as follows:

Proposition 2. At no time to the past of a cusp on $H$ will the surface of a black hole be without cusps. 
It is sometimes suggested that caustics just exist for some finite time interval during which the actual processes take place, like collision and coalescence of black holes or the infall of matter through the horizon (see e.g. [MTW] 34.5). Proposition 2 shows that this is not quite the right picture.

\section{References}

[BK] J.K. Beem and A. Królak: Cauchy horizon endpoints and differentiability. gr-qc/9709046

[CG] P.T. Chruściel and G.J. Galloway: Horizons non-differentiable on a dense set. Comm. Math. Phys. 193 (1998), 449-470.

[D] A. Dold: Lectures on algebraic topology, Springer Verlag, Berlin, 1972.

[F] H. Federer: Geometric measure theory, Springer Verlag, New York, 1969.

[HE] S.W. Hawking and G.F.R. Ellis: The large scale structure of space-time, Cambridge University Press, 1973.

[L] S. Lang: Differential Manifolds, Springer-Verlag, Berlin, 1985.

[L-W] J. Libson, J. Massó, E. Seidel, W.-M. Suen and P. Walker: Event horizons in numerical relativity: Methods and tests. Phys. Rev. D 53 (1996), 4335-4350.

[MTW] C.W. Misner, K.S. Thorne and J.A. Wheeler: Gravitation, W.H. Freeman and Company, San Francisco, 1973.

[W] R.M. Wald: General Relativity, The University of Chicago Press, Chicago and London, 1984. 